\title{
Sensibilidade e especificidade de diferentes critérios de classificação do excesso de peso em escolares de João Pessoa, Paraíba, Brasil
}

\author{
Sensitivity and specificity of different classification \\ criteria for excess weight in schoolchildren \\ from João Pessoa, Paraíba, Brazil
}

Kelly Samara da SILVA ${ }^{2}$

Adair da Silva LOPES 3

Francisco Martins da SILVA ${ }^{4}$

\section{R E S U M O}

\section{Objetivo}

Descrever a proporção de excesso de peso usando diferentes critérios de classificação do índice de massa corporal e avaliar valores de sensibilidade, especificidade e concordância entre os critérios.

\section{Métodos}

A população incluiu 1570 estudantes, de 7 a 12 anos de idade, da cidade de João Pessoa (PB), 2005. O excesso de peso foi classificado conforme os critérios da World Health Organization, da International Obesity Task Force e por outros autores. O percentual de gordura foi estimado a partir das dobras cutâneas e utilizado como critério de referência (masculino: $\geq 25 \%$; feminino: $\geq 30 \%$ ).

\section{Resultados}

A proporção de excesso de peso diferiu entre os critérios, sendo menor com a utilização dos critérios da World Health Organization e maior segundo o critério de autores brasileiros $(p<0,05)$. Os critérios de classificação do índice de massa corporal apresentaram boa sensibilidade (83-97\%), exceto o critério da World Health Organization (masculino=65\% e feminino=48\%). A especificidade foi elevada em todos os valores críticos analisados (85-98\%). O índice Kappa demonstrou boa concordância entre as propostas (Kappa >0,60), com

1 Artigo elaborado a partir da dissertação de K.S. SILVA, intitulada "Sedentarismo, excesso de peso corporal e pressão arterial elevada em crianças e adolescentes de João Pessoa, PB”. Universidade Federal de Santa Catarina; 2007.

${ }^{2}$ Universidade Federal da Paraíba, Centro de Ciências da Saúde, Departamento de Educação Física. Campus I, Cidade Universitária, s/n., 58059-900, João Pessoa, PB, Brasil. Correspondência para/Correspondence to: K.S. SILVA. E-mail: $<$ ksilvajp@yahoo.com.br>.

3 Universidade Federal de Santa Catarina, Centro de Desportos, Programa de Pós-Graduação em Educação Física. Florianópolis, SC, Brasil.

${ }^{4}$ Universidade Católica de Brasília, Programa de Pós-Graduação em Educação Física. Brasília, DF, Brasil. 
discreto aumento no critério da IOTF (Kappa=0,72) e menor concordância para a proposta da World Health Organization (Kappa=0,63).

\section{Conclusão}

Os critérios analisados foram sensíveis para diagnosticar o excesso de peso, com menor sensibilidade ao ser adotada a proposta da World Health Organization. Houve elevada especificidade e boa concordância em todas as propostas, entretanto, os valores para os sexos e as idades oscilaram menos no critério da International Obesity Task Force. Portanto, os resultados sugerem que o critério da International Obesity Task Force mostrou-se mais adequado para confirmar a presença de excesso de peso em populações com características similares às deste estudo.

Termos de indexação: Avaliação do estado nutricional. Dobras cutâneas. Índice de massa corporal. Medidas antropométricas. Sensibilidade e especificidade. Sobrepeso.

\section{A B S T R A C T}

\section{Objective}

The objective of this study was to describe the proportion of excess weight using different body mass index classification criteria and assess the sensitivity, specificity, and agreement among the criteria.

\section{Methods}

The population included 1,570 students, aged 7-12 years, of the city of João Pessoa (PB), in 2005. Excess weight was classified according to the World Health Organization, International Obesity Task Force criteria and other criteria. The percentage of body fat was estimated from skinfold thickness measurements and used as reference criteria ( $\geq 25 \%$ in males and $\geq 30 \%$ in females).

\section{Results}

The proportion of excess weight differed among the different classification systems. It was lower according to the World Health Organization criteria than to Brazilian authors' criteria $(p<0.05)$. Body mass index classification criteria presented high sensitivity (83-97\%), except for the classification proposed by the World Health Organization (65\% in males and $48 \%$ in females). The specificity was high for all the analyzed criteria (85-98\%). The Kappa index showed good agreement among the criteria, with a small increase for the International Obesity Task Force criteria (Kappa=0.72) and smaller agreement for the World Health Organization criteria (Kappa=0.63).

\section{Conclusion}

The analyzed criteria were sensitive for the detection of excess weight, but the World Health Organization criteria were less sensitive. There was high specificity and agreement among the criteria; however, the values obtained for the different genders and ages varied less when the International Obesity Task Force criteria were used. Thus, the results suggest that the International Obesity Task Force criteria are more appropriate for the determination of excess weight in similar populations.

Indexing terms: Evaluate nutritional status. Skinfold thickness. Body mass index. Anthropometric measures. Sensitivity and specificity. Overweight.

\section{N T R O D U ÇÃ O}

A relevância em investigar a precisão de parâmetros de classificação de excesso de peso em jovens é importante, por auxiliar na acurácia do diagnóstico, no controle e no acompanhamento de programas de intervenção. Segundo a World Health Organization (WHO)' 1 , o uso do Índice de Massa Corporal (IMC) é apropriado por apresentar boa correlação com a gordura corporal, ser de fácil obtenção e permitir comparações com outros estudos. Porém, a determinação de um referencial do estado nutricional é complexa, visto que diferenças populacionais e a ausência de validação entre os critérios têm dificultado a escolha. Logo, o desafio atual consiste em selecionar o critério de referência mais adequado, tendo em vista parâmetros internacionais ${ }^{2-4}$ e nacionais ${ }^{5}$. 
Estudos internacionais ${ }^{5-11}$ e nacionais ${ }^{12-15}$ observaram que o IMC foi adequado para mensurar excesso de peso em crianças $^{16-19}$. As principais propostas de classificação de IMC difundidas em pesquisas brasileiras são os valores críticos sugeridos pela $\mathrm{WHO}^{2}$, pela International Obesity Task Force (IOTF) ${ }^{3}$, por Must et al. ${ }^{4}$ e, recentemente, os valores sugeridos por Conde \& Monteiro ${ }^{5}$. O uso de curvas percentílicas para diagnosticar excesso de peso tem recebido críticas em função de os pontos de corte demarcar, inicialmente, um número aparente de crianças com o desfecho ${ }^{2,4}$. Por outro lado, os critérios que recorrem a valores fixos também sofrem críticas quanto à utilização em programas de saúde pública e de prática clínica ${ }^{3,5}$.

Ainda não há um consenso entre os estudos quanto à escolha do critério de classificação do IMC e pesquisadores têm se preocupado com a precisão de diagnóstico dos parâmetros existentes. Alguns estudos, $6,7,12-15$ revelaram melhor predição de excesso de peso, utilizando a proposta da IOTF$^{3}$, de Must et al. ${ }^{4}$ ou de critérios brasileiros ${ }^{5,20}$. Nesse sentido, o presente estudo teve os seguintes objetivos: descrever o perfil antropométrico e investigar a correlação entre o IMC, as dobras cutâneas e a gordura corporal; estimar a proporção de excesso de peso utilizando quatro critérios de classificação do IMC e avaliar os valores de sensibilidade, especificidade e concordância destes.

\section{MÉTODOS}

Este estudo é parte de um levantamento epidemiológico, de corte transversal, sobre a prevalência e os fatores associados a doenças cardiovasculares em estudantes da cidade de João Pessoa (PB). A pesquisa foi desenvolvida entre abril e setembro de 2005, após aprovação do Comitê de Ética em Pesquisa, envolvendo seres humanos da Universidade Federal da Paraíba (RG 129/03).

A população incluiu estudantes do Ensino Fundamental, de 7 a 12 anos de idade, de ambos os sexos. O processo amostral foi estratificado pela divisão da cidade em cinco distritos, conforme dados da Secretaria de Saúde do Município, e dividido em dois estágios: 1) Escolas (Públicas Municipais e Particulares) e 2) Turmas (de $1^{\mathrm{a}}$ à $4^{\mathrm{a}}$ série). Dos 248 estabelecimentos de Ensino Fundamental existentes no Município, foram excluídos aqueles freqüentados apenas por meninas $(n=10)$ e os que não apresentavam todas as séries, ou que possuíam um número de alunos inferior a 30 $(n=88)$. Das 150 escolas consideradas elegíveis, selecionaram-se 15 (10 municipais e 5 particulares), sendo avaliadas 3 escolas em cada distrito.

O procedimento da amostra foi aleatório por conglomerados (turmas intactas), e para o cálculo amostral ${ }^{21}$, como outras variáveis foram coletadas, considerou-se a estimativa de prevalência referente ao maior desfecho $(60 \%$ de sedentarismo) $)^{22}$, intervalo de confiança de $95 \%$, erro tolerável de 3\% e 1,5 para efeito de desenho. Com isso, estimou-se um tamanho de amostra mínima de 1497 escolares e, por segurança, decidiu-se acrescentar $10 \%$, para compensar eventuais perdas. Em conformidade com o plano amostral, 1647 estudantes, aparentemente saudáveis e livres de tratamento médico participaram do estudo.

Foram incluídos todos os estudantes, com idades de 7 a 12 anos, que entregaram o termo de consentimento, assinado por seus responsáveis, e excluídos aqueles fora da faixa etária $(n=60$; $3,6 \%)$. Houve $1,0 \%(n=17)$ de perda e recusa entre os estudantes que faltaram no dia do teste, ou que se recusaram a realizar as medidas. A amostra foi de 1570 (808 meninos e 762 meninas), mantendo-se o poder estatístico inicial e a representatividade da população.

Na avaliação antropométrica, recorreu-se à medida de massa corporal e de estatura, seguindo normas padronizadas ${ }^{23}$. Para aferir a massa corporal $(\mathrm{kg})$, utilizou-se uma balança digital da marca Plenna, admitindo-se variação mínima de $0,1 \mathrm{~kg}$ entre duas medidas. A estatura $(\mathrm{cm})$ foi aferida com uma fita métrica, com escalas de $0,1 \mathrm{~cm}$, fixada à parede nivelada, admitindo-se 
uma variação de $0,5 \mathrm{~cm}$ entre duas medidas. As Dobras Cutâneas Triciptal (TR) e Subescapular (SE) foram mensuradas por meio de um plicômetro modelo Harpenden, sendo registrado o valor médio de duas medidas, obtidas com intervalo de dois minutos. As dobras foram aferidas por dois avaliadores experientes com erro técnico de medida $^{24}$ intra e inter-avaliador inferior a $2 \%$ e coeficiente de correlação intra-classe de 0,97 $\left(I C_{95 \%}=0,95-0,98\right)$.

O IMC foi utilizado para classificar o excesso de peso, segundo quatro critérios: $\mathrm{WHO}^{2} \mathrm{e}$ Must et a/4. para valores de IMC no percentil $\geq 85$, e IOTF $^{3}$ e Conde \& Monteiro 5 para valores de IMC $\geq 25 \mathrm{~kg} / \mathrm{m}^{2}$. A adiposidade corporal (referência) foi definida com base no percentual de Gordura (\%GC), usando as equações propostas por Boileau et al. ${ }^{25} \mathrm{e}$ os pontos de corte sugeridos por Willians et al. 26 (\%GC $\geq 25 \%$ para o sexo masculino e $30 \%$ para o sexo feminino).

1) Masculino: $\% G C=1,35\left(\sum D C\right)-0,012$ $\left(\sum D C\right)^{2}-4,4$

2) Feminino: $\% G C=1,35\left(\sum D C\right)-0,012$ $(\Sigma D C)^{2}-2,4$

As diferenças de média do IMC e das dobras cutâneas entre os sexos para a mesma faixa etária e entre as idades para cada sexo foram avaliadas por meio da Análise de Variância (ANOVA) e do teste post hoc de Scheffe $(p<0,05)$. A correlação entre as medidas de dobras cutâneas e do IMC foi obtida usando a correlação de Pearson. As diferenças de prevalências de excesso de peso entre os critérios analisados, considerando o sexo e a idade, foram avaliadas mediante o teste de McNemar.

Quanto à adiposidade, adotou-se o percentual de gordura corporal como padrão de referência, para cálculo de sensibilidade (Sens) e de especificidade (Espec). A sensibilidade do teste foi definida como a probabilidade das respectivas propostas em detectar os indivíduos com excesso de gordura corporal (verdadeiro-positivos). A especificidade consistiu na probabilidade de distinguir os indivíduos sem excesso de gordura corporal (verdadeiro-negativos).
Para avaliar a curva Receiver Operator Characteristic (ROC), os escolares foram divididos em duas faixas etárias: 7 a 9 anos e 10 a 12 anos'. A área sob a curva foi utilizada para caracterizar o IMC, com base no percentual de gordura. A curva ROC descreve o desempenho do teste, em termos do diagnóstico preciso, para classificar adequadamente os indivíduos. O nível de concordância entre os quatro critérios foi estimado por meio do Coeficiente Kappa, e o nível de significância para todas as análises foi menor que 0,05 $(p<0,05)$.

\section{RES U L T A D OS}

As médias das variáveis antropométricas diferiram estatisticamente $(p<0,05)$ entre os sexos (dobra cutânea triciptal - TR - e soma das dobras cutâneas triciptal e subescapular - $\Sigma D C$ ) e entre as idades (todas as variáveis). O peso e a estatura aumentaram com o aumento da idade e a média da dobra subescapular (SE) foi maior entre os escolares de 10 e 11 anos. No sexo feminino, as médias do IMC, da dobra TR e da $\Sigma D C$ foram menores entre as estudantes de 7-8 anos, em comparação às de 10-12 anos. No sexo masculino, a média da dobra TR foi menor aos 12 anos, em relação aos 10 e 11 anos (Tabela 1).

Houve correlação positiva $(p<0,05)$ entre o IMC e a $\Sigma D C$ (masculino: $r=0,88$; feminino: $r=0,85$ ), entre o IMC e a dobra TR (masculino: $r=0,86$; feminino: $r=0,79$ ) e a dobra SE (masculino: $r=0,85$; feminino: $r=0,83$ ). Também houve forte correlação $(p<0,05)$ entre o percentual de gordura e o IMC (masculino: $r=0,86$; feminino: $r=0,85$ ), a $\sum D C$ (masculino: $r=0,91$; feminino: $r=0,95$ ), a dobra TR (masculino: $r=0,93$; feminino: $r=0,93$ ) e a dobra SE (masculino: $r=0,84$; feminino: $r=0,88)$. Porém, a elevada correlação não garante a validade clínica dos critérios de classificação.

Segundo o critério da $\mathrm{WHO}^{2}$ houve menor proporção de excesso de peso nos escolares de 7 a 10 anos de idade, em relação ao critério da IOTF $^{3}$ e ao de Must et al. ${ }^{4}$, e diferiu do de Conde \& Monteiro ${ }^{5}$, até os 11 anos $(p<0,05)$. Os resulta- 
Tabela 1. Valores médios e desvios-padrão dos indicadores antropométricos de escolares, por sexo e idade. João Pessoa (PB), 2005.

\begin{tabular}{|c|c|c|c|c|c|c|c|c|c|c|c|c|}
\hline \multirow{2}{*}{ Sexo e Idade } & \multicolumn{2}{|c|}{ Peso } & \multicolumn{2}{|c|}{ Estatura } & \multicolumn{2}{|c|}{ IMC } & \multicolumn{2}{|c|}{ TR } & \multicolumn{2}{|c|}{ SE } & \multicolumn{2}{|c|}{$\Sigma D C$} \\
\hline & $\mathrm{M}$ & $\mathrm{DP}$ & $M$ & $\mathrm{DP}$ & $\mathrm{M}$ & $\mathrm{DP}$ & $M$ & $\mathrm{DP}$ & $M$ & $\mathrm{DP}$ & $M$ & $\mathrm{DP}$ \\
\hline \multicolumn{13}{|l|}{ Masculino } \\
\hline 7 & 25,4 & 5,7 & 1,2 & 6,3 & 16,5 & 2,9 & 11,4 & 5,1 & 8,7 & 5,9 & 20,2 & 10,8 \\
\hline 8 & 28,3 & 6,8 & 1,3 & 6,3 & 16,9 & 3,0 & 11,9 & 5,9 & 9,3 & 6,5 & 21,2 & 12,2 \\
\hline 9 & 29,4 & 6,4 & 1,3 & 7,1 & 16,6 & 2,5 & 11,6 & 5,0 & 8,7 & 5,6 & 20,3 & 10,3 \\
\hline 10 & 33,5 & 8,3 & 1,4 & 6,9 & 17,5 & 3,1 & 12,9 & 6,1 & 10,0 & 7,1 & 23,0 & 12,7 \\
\hline 11 & 35,7 & 7,7 & 1,4 & 6,7 & 17,7 & 3,3 & 12,8 & 5,8 & 10,1 & 7,2 & 22,8 & 12,6 \\
\hline 12 & 36,5 & 7,4 & 1,5 & 5,8 & 17,1 & 2,5 & 10,2 & 3,7 & 8,3 & 4,6 & 18,5 & 8,1 \\
\hline Total & 31,3 & 7,9 & 1,4 & 9,1 & 17,1 & 2,9 & 12,1 & 5,6 & 9,3 & 6,4 & 21,4 & 11,7 \\
\hline \multicolumn{13}{|l|}{ Feminino } \\
\hline 7 & 24,3 & 4,5 & 1,2 & 4,6 & 16,3 & 2,4 & 11,9 & 4,3 & 9,5 & 5,1 & 21,3 & 9,1 \\
\hline 8 & 26,4 & 5,5 & 1,3 & 6,2 & 16,4 & 2,6 & 12,7 & 4,8 & 9,7 & 5,6 & 22,4 & 10,0 \\
\hline 9 & 30,2 & 6,6 & 1,3 & 7,0 & 16,9 & 2,6 & 13,2 & 4,6 & 10,3 & 5,3 & 23,4 & 9,4 \\
\hline 10 & 32,6 & 7,8 & 1,4 & 7,1 & 17,1 & 2,9 & 14,2 & 5,2 & 11,4 & 7,0 & 25,6 & 11,5 \\
\hline 11 & 37,3 & 9,5 & 1,4 & 8,4 & 17,9 & 3,2 & 14,7 & 5,4 & 12,1 & 7,2 & 26,8 & 12,2 \\
\hline 12 & 40,4 & 8,9 & 1,5 & 8,7 & 18,1 & 2,9 & 14,4 & 4,9 & 10,8 & 5,0 & 25,2 & 9,5 \\
\hline Total & 30,9 & 8,5 & 1,3 & 0,1 & 16,9 & 2,8 & 13,4 & 4,9 & 10,6 & 6,1 & 24,1 & 10,5 \\
\hline Fidade & \multicolumn{2}{|c|}{$81,88^{*}$} & \multicolumn{2}{|c|}{$239,15^{*}$} & \multicolumn{2}{|c|}{$8,51^{*}$} & \multicolumn{2}{|c|}{$7,34^{*}$} & \multicolumn{2}{|c|}{$5,91^{*}$} & \multicolumn{2}{|c|}{$7,05^{*}$} \\
\hline F sexo & \multicolumn{2}{|c|}{0,57} & \multicolumn{2}{|c|}{0,84} & \multicolumn{2}{|c|}{0,06} & \multicolumn{2}{|c|}{$6,57^{*}$} & \multicolumn{2}{|c|}{2,82} & \multicolumn{2}{|c|}{$4,68^{*}$} \\
\hline F sexolidade & \multicolumn{2}{|c|}{$2.23^{*}$} & \multicolumn{2}{|c|}{$4.07^{*}$} & \multicolumn{2}{|c|}{$0,61^{*}$} & \multicolumn{2}{|c|}{0.67} & \multicolumn{2}{|c|}{0,99} & \multicolumn{2}{|c|}{0,87} \\
\hline
\end{tabular}

* teste pós Roc (Receiver Operator Characteristic) de Scheffé com nível de significância menor que 0,05.

IMC: índice de massa corporal; TR: dobra cutânea triciptal; SE: dobra cutânea subescapular; LDC: soma das dobras cutâneas triciptal e subescapular; M: média; DP: desvios-padrão; F: análise de variância Two Way.

Tabela 2. Proporções de excesso de peso de escolares, segundo diferentes critérios de referência do índice de massa corporal, por sexo e idade. João Pessoa (PB), 2005.

\begin{tabular}{|c|c|c|c|c|c|c|c|c|}
\hline \multirow{2}{*}{ Sexo e Idade } & \multicolumn{8}{|c|}{ Critério de classificação do IMC } \\
\hline & $\%$ & (IC 95\%) & $\%$ & (IC 95\%) & $\%$ & (IC 95\%) & $\%$ & (IC 95\%) \\
\hline \multicolumn{9}{|l|}{ Masculino } \\
\hline 7 & 10,6 & $(3,9-17,3)$ & 28,2 & $(19,7-\underline{0} 8,5)$ & 30,6 & $(21,8-41,0)$ & 29,4 & $(20,7-39,8)$ \\
\hline 8 & 13,0 & $(7,5-18,5)$ & 21,9 & $(15,9-29,3)$ & 23,3 & $(17,2-30,8)$ & 21,9 & $(15,9-29,3)$ \\
\hline 10 & 16,2 & $(10,8-21,7)$ & 19,0 & $(13,9-25,4)$ & 21,3 & $(15,9-27,9)$ & 24,6 & $(18,9-31,4)$ \\
\hline 11 & 19,6 & $(13,0-26,2)$ & 19,6 & $(13,9-26,9)$ & 22,5 & $(16,4-30,0)$ & 24,5 & $(18,2-32,2)$ \\
\hline 12 & 9,8 & $(1,4-18,3)$ & 7,8 & $(3,1-18,4)$ & 7,8 & $(3,1-18,4)$ & 9,8 & $(4,3-21,0)$ \\
\hline Total & 12,9 & $(10,6-15,3)$ & 19,4 & $(16,8-22,3)$ & 21,9 & $(19,2-24,9)$ & 22,4 & $(19,7-25,4)$ \\
\hline \multicolumn{9}{|l|}{ Feminino } \\
\hline 10 & 9,7 & $(5,3-14,1)$ & 19,9 & $(14,7-26,4)$ & 19,5 & $(14,3-25,1)$ & 27,3 & $(21,3-34,3)$ \\
\hline 11 & 14,6 & $(7,6-21,5)$ & 16,5 & $(10,6-24,8)$ & 15,8 & $(10,0-24,1)$ & 25,2 & $(17,8-34,4)$ \\
\hline 12 & 13,7 & $(4,0-23,5)$ & 13,7 & $(6,8-25,7)$ & 11,8 & $(5,5-23,4)$ & 19,6 & $(11,0-32,5)$ \\
\hline Total & 8,7 & $(6,7-10,8)$ & 20,4 & $(17,7-23,4)$ & 22,4 & $(19,6-25,5)$ & 27,2 & $(24,1-30,5)$ \\
\hline
\end{tabular}

WHO: World Health Organization; IOTF: International Obesity Task Force; IC 95\%: intervalo de confiança de $95 \%$. 
dos utilizando os parâmetros do IOTF $^{3}$ diferiram daqueles do critério de Must et al. ${ }^{4}$ nos meninos de 9 anos e nas meninas de 7 e 8 anos, e do de Conde \& Monteiro ${ }^{5}$ nos meninos de 10 e 11 anos e nas meninas de 8 a 11 anos $(p<0,05)$. De acordo com o critério de Conde \& Monteiro ${ }^{5}$ houve maior proporção de excesso de peso nas meninas de 9 a 11 anos de idade, em relação ao de Must et al. ${ }^{4}(p<0,05)$ (Tabela 2).

Os valores de área sob a curva $R O C$ demonstraram que o IMC foi um forte indicador de adiposidade corporal (Tabela 3). Os critérios analisados apresentaram boa sensibilidade (83\% - 97\%), exceto a proposta da $\mathrm{WHO}^{2}$, que deixou de de- tectar 35,5\% dos meninos e 52,3\% das meninas com excesso de peso. A especificidade foi elevada em todos os critérios analisados (85\% - 98\%), com destaque para a proposta da IOTF ${ }^{3}$, que foi mais específica e menos sensível que a proposta de Conde \& Monteiro ${ }^{5}$, não diagnosticando 14,2\% dos estudantes que apresentavam excesso de peso. Na proposta de Must et al. ${ }^{4}$ houve um declínio da sensibilidade, em ambos os sexos, com o aumento da idade (Tabela 4).

O índice Kappa demonstrou boa concordância entre os quatro critérios de classificação do excesso de peso, quando comparados ao padrão-ouro, entretanto, observou-se resultados

Tabela 3. Área sob a Curva ROC (Receiver Operator Characteristic) para o uso do índice de massa corporal, em relação ao percentual de gordura corporal de escolares. João Pessoa (PB), 2005.

\begin{tabular}{|c|c|c|c|c|c|c|}
\hline \multirow{2}{*}{ Sexo Idade (anos) } & \multicolumn{3}{|c|}{ Masculino } & \multicolumn{3}{|c|}{ Feminino } \\
\hline & Área & IC 95\% & EP & Área & IC 95\% & $E P$ \\
\hline 7 a 9 & 0,98 & $0,97-0,99$ & 0,007 & 0,97 & $0,95-0,98$ & 0,008 \\
\hline 10 a 12 & 0,97 & $0,95-0,99$ & 0,008 & 0,96 & $0,95-0,98$ & 0,009 \\
\hline Total & 0,97 & $0,96-0,98$ & 0,005 & 0,97 & $0,95-0,98$ & 0,006 \\
\hline
\end{tabular}

Área: valor da área sob da curva ROC; IC 95\%: intervalo de confiança de 95\%; EP: erro padrão obtido na curva ROC.

Tabela 4. Sensibilidade e especificidade dos critérios de classificação do índice de massa corporal de escolares, em relação ao percentual de gordura estimado a partir das dobras cutâneas. João Pessoa (PB), 2005.

\begin{tabular}{|c|c|c|c|c|c|c|c|c|}
\hline \multirow{3}{*}{ Sexo e Idade } & \multicolumn{8}{|c|}{ Critério de classificação do IMC } \\
\hline & \multicolumn{2}{|c|}{ WHO } & \multicolumn{2}{|c|}{ IOTF $^{3}$} & \multicolumn{2}{|c|}{ Must et al. ${ }^{4}$} & \multicolumn{2}{|c|}{ Conde \& Monteiro ${ }^{5}$} \\
\hline & Sens & Espec & Sens & Espec & Sens & Espec & Sens & Espec \\
\hline \multicolumn{9}{|l|}{ Masculino } \\
\hline 7 & 57,1 & 98,6 & 100,0 & 85,9 & 100,0 & 83,1 & 100,0 & 84,5 \\
\hline 8 & 66,7 & 99,1 & 96,3 & 94,9 & 96,3 & 93,2 & 96,3 & 94,5 \\
\hline 9 & 44,8 & 99,4 & 86,2 & 95,2 & 92,9 & 91,6 & 89,7 & 92,9 \\
\hline 10 & 73,5 & 97,2 & 85,3 & 96,6 & 88,2 & 94,4 & 88,2 & 90,3 \\
\hline 11 & 74,2 & 95,5 & 74,2 & 95,5 & 80,6 & 93,7 & 87,1 & 92,9 \\
\hline 12 & 75,0 & 95,7 & 75,0 & 97,9 & 75,0 & 97,9 & 75,0 & 95,7 \\
\hline Total & 64,7 & 97,9 & 86,3 & 94,7 & 89,9 & 92,4 & 90,6 & 92,0 \\
\hline \multicolumn{9}{|l|}{ Feminino } \\
\hline 7 & 30,0 & 100,0 & 100,0 & 81,9 & 100,0 & 71,1 & 100,0 & 77,1 \\
\hline 8 & 62,5 & 100,0 & 93,8 & 87,0 & 93,8 & 82,4 & 93,8 & 80,2 \\
\hline 9 & 36,0 & 96,8 & 92,0 & 91,1 & 92,0 & 89,8 & 100,0 & 84,1 \\
\hline 10 & 41,4 & 96,6 & 89,7 & 93,9 & 86,2 & 93,8 & 100,0 & 87,1 \\
\hline 11 & 57,1 & 96,3 & 61,9 & 95,1 & 60,0 & 95,1 & 95,2 & 92,7 \\
\hline 12 & 75,0 & 97,7 & 75,0 & 97,7 & 62,5 & 97,7 & 87,5 & 93,0 \\
\hline Total & 47,7 & 97,8 & 85,3 & 90,7 & 83,3 & 88,0 & 97,2 & 84,8 \\
\hline
\end{tabular}

WHO: World Health Organization; IOTF: International Obesity Task Force; Sens: sensibilidade; Espec: especificidade. 
Tabela 5. Concordância entre os critérios para a classificação do excesso de peso corporal de escolares, em comparação com o percentual de gordura. João Pessoa (PB), 2005

\begin{tabular}{lllcc}
\hline \multirow{2}{*}{ Sexo } & \multicolumn{4}{l}{ Percentual de gordura corporal } \\
\cline { 2 - 5 } & WHO $^{2}$ & IOTF & Must et al. ${ }^{4}$ & Conde \& Monteiro $^{5}$ \\
\hline Masculino & 0,695 & 0,775 & 0,746 & 0,742 \\
Feminino & 0,544 & 0,651 & 0,581 & 0,602 \\
\hline Total & 0,631 & 0,716 & 0,668 & 0,672 \\
\hline
\end{tabular}

WHO: World Health Organization; IOTF: International Obesity Task Force.

Classificação do teste de Concordância Kappa: muito bom =0,81-1,00; bom =0,61-0,80; moderado =0,41-0,60; débil =0,21-0,40 e insuficiente $<0,20$.

próximos quando adotados os critérios de Conde \& Monteiro ${ }^{5}$ e de Must et al. ${ }^{4}$ (Kappa=0,67), menor concordância na proposta da $\mathrm{WHO}^{2}$ (Kappa=0,63) e maior concordância na proposta da IOTF$^{3}$ (Kappa=0,72) (Tabela 5).

\section{DIS CUSS Ã O}

Houve forte correlação do IMC $(r=0,86)$ com as dobras cutâneas, em ambos os sexos. Resultados similares foram encontrados em estudos que usaram a dobra subescapular ${ }^{12}$ ou o percentual de gordura estimado pelo DEXA ${ }^{6-10,13}$, como padrão-ouro. Pesquisas com crianças americanas de 6-11 anos ${ }^{8}$ e portuguesas de 10-11 anos de idade $^{16}$, também revelaram que o IMC foi um forte preditor de adiposidade, quando comparado ao percentual de gordura.

Apesar da boa correlação do IMC com a gordura corporal, um estudo ${ }^{17}$ demonstrou que, para um dado ponto de corte do IMC, ocorrem aumentos transitórios na adiposidade entre os meninos e aumento progressivo com a idade, entre as meninas, antes de iniciar o platô ( $>12$ anos). Em escolares austríacos ${ }^{18}$ o IMC explicou $73 \%$ da variação do percentual de gordura nos meninos de 6 a 10 anos de idade, e somente $27 \%$ naqueles com 11-17 anos. 0 mesmo foi observado nas meninas, com $63 \%$ e $38 \%$, respectivamente. Os autores concluíram que, para o mesmo sexo, idade e IMC foram encontradas escalas de valores diferentes para o percentual de gordura, o que indica que a associação do IMC à gordura, somente é sensível e confiável em crianças pré-púberes ${ }^{16-19}$.
Outra dificuldade para o uso do IMC é a escolha da referência e do ponto de corte adequado, para identificar e monitorar o excesso de peso em crianças. No presente estudo, o critério de Conde \& Monteiro ${ }^{5}$ apresentou prevalências de 5 a 18 pontos percentuais mais elevadas para as meninas e a proposta da $\mathrm{WHO}^{2}$ apresentou prevalências mais baixas, em ambos os sexos, quando comparados aos valores encontrados nas demais propostas. Os resultados sugerem que o critério da $\mathrm{WHO}^{2}$ subestima as prevalências de excesso de peso em ambos os sexos e a proposta de Conde \& Monteiro superestima para o sexo feminino. Outras pesquisas ${ }^{13-15}$ também observaram maior risco de sobrepeso, quando usadas as curvas brasileiras, em relação à da $\operatorname{IOTF}^{3}$ ou à de Must et al. ${ }^{4}$.

No presente estudo, O IMC apresentou boa condição de diagnóstico da adiposidade corporal, com elevada área sob a curva ROC. O critério de Conde \& Monteiro ${ }^{5}$ apresentou maior poder de diagnóstico do excesso de peso, reduzindo o número de casos falso-negativos, mas apresentou menor capacidade de reduzir os casos falso-positivos nas meninas. A proposta da $\mathrm{WHO}^{2}$ demonstrou elevada capacidade de distinguir os indivíduos sem excesso de peso, diminuindo os casos falso-positivos, entretanto, revelou baixo poder de diagnóstico do excesso de peso, em comparação aos demais critérios, principalmente na faixa etária de 7 a 10 anos. A proposta da IOTF $^{3}$ demonstrou elevada especificidade e boa sensibilidade, e o critério de Must et al. ${ }^{4}$ apresentou bons parâmetros para o sexo masculino.

Segundo Onis et al. ${ }^{27}$, na construção das curvas da $\mathrm{WHO}^{2}$ de IMC para idade foram consi- 
derados dados de referência do relatório de 1991, em que haviam informações disponíveis somente a partir dos 9 anos de idade. Com isso, as curvas abaixo desta idade foram estimadas por meio de cálculos estatísticos para a reconstrução das tabelas de 2007, que permitiram a extensão da referência do IMC para idades menores. Sabe-se que parte da variabilidade na sensibilidade das propostas de classificação do IMC pode ser influenciada pela estimativa de valores estatísticos e por condições sócio-demográficas e ambientais ${ }^{6}$.

Estudos que envolvem adolescentes mais velhos não têm observado diferenças significativas entre esses critérios ${ }^{28,29}$. Também foi observada baixa sensibilidade entre as propostas em diagnosticar adequadamente a adiposidade no sexo feminino, chegando a classificar erroneamente de $40 \%$ a $50 \%$ de adolescentes no Rio de Janeiro ${ }^{15}$ e de $58 \%$ a $63 \%$ em Florianópolis ${ }^{28}$, e ainda, $75 \%$ de adolescentes suecas ${ }^{6}$ e $50 \%$ de crianças suíças ${ }^{7}$. Diversos estudos nacionais ${ }^{13-15}$ revelaram maior sensibilidade para o excesso de peso, quando usados os critérios brasileiros. Neste estudo, observouse boa concordância entre as propostas analisadas, com discreto aumento nos valores críticos propostos pela IOTF ${ }^{3}$. Resultados similares foram observados em crianças mexicanas (6-10 anos) ${ }^{30}$.

Embora existam diferenças metodológicas entre as pesquisas analisadas e o presente estudo, os resultados foram qualitativamente similares e se especula o uso do IMC na predição da adiposidade em crianças pré-púberes, porém, nas crianças púberes, sugere-se o controle de outros indicadores de gordura, pela vulnerabilidade do IMC nesse grupo. Dentre as limitações, destacam-se o uso de método duplamente indireto como padrão-ouro e a comparação entre critérios que adotaram curvas percentílicas e cortes fixos de IMC. Todavia, sabe-se que o percentual de gordura corporal mantém valores de adiposidade próximos aos de medidas mais precisas.

Os dados deste estudo revelaram maior adiposidade entre as meninas e correlação positiva entre o IMC e as dobras cutâneas para ambos os sexos. As propostas analisadas apresentaram bom índice de concordância, com destaque para o critério da $\mathrm{IOTF}^{3}$ e menor precisão para o critério da $\mathrm{WHO}^{2}$. Os resultados sugerem que o critério da IOTF mostrou-se mais adequado para confirmar a presença de excesso de peso e evitar intervenções e tratamentos desnecessários em crianças com características similares a este estudo.

\section{COLABORADORES}

K.S. SILVA foi responsável pela estruturação do projeto, pela coleta de dados e pela elaboração do manuscrito. A.S. LOPES participou da elaboração e da revisão crítica do artigo. F.M. SILVA participou da construção e da análise crítica do manuscrito.

\section{REFERÊ N CIAS}

1. World Health Organization. Physical status: the use and interpretation of anthropometry. Geneva: WHO;1995. Report of a WHO Expert Committee.

2. World Health Organization. Growth reference data for 5-19 years. 2007 [cited 2009 Abr 16]. Available from: <http://www.who.int/growthref/en/>.

3. Cole TJ, Bellizzi MC, Flegal KM, Dietz WH. Establishing a standard definition for child overweight and obesity worldwide: international survey. BMJ. 2000; 6;320(7244):1240-3. doi: 10.1136/bmj.320.7244.1240.

4. Must A, Dallal GE, Dietz WH. Reference data for obesity: $85^{\text {th }}$ and $95^{\text {th }}$ percentiles of body mass index (wt/ht2) and triceps skinfold thikness. Am J Clin Nutr. 1991; 53(4):839-46.

5. Conde WL, Monteiro CA. Valores críticos do índice de massa corporal para classificação do estado nutricional de crianças e adolescentes brasileiros. J Pediatr. 2006; 82(4):266-72. doi: 10.2223/jped. 1502.

6. Neovius M, Linné $Y$, Barkeling B, Rossner S. Sensitivity and specificity of classification systems for fatness in Adolescents. Am J Clin Nutr. 2004; 80(3):597-603.

7. Zimmermann MB, Gubeli C, Puntener C, Molinari L. Detection of overweight and obesity in a national sample of 6-12y old Swiss children: accuracy and validity of reference values for body mass index from the US Centers for Disease Control and Prevention and the International Obesity Task Force. Am J Clin Nutr. 2004; 79(5):838-43.

8. Mei Z, Grummer-Strawn LM, Pietrobelli A, Goulding A, Goran IM, Dietz HW. Validity of body mass index compared with other body-composition 
screening indexes for the assessment of body fatness in children and adolescents. Am J Clin Nutr. 2002; 75(6):978-85.

9. Watts K, Naylor LH, Davis EA, Jones TW, Beeson B, Bettenay $F$, et al. Do Skinfolds accurately assess changes in body fat in obese children and adolescents? Med Sci Sports Exerc. 2006; 38(3): 439-44.

10. Semiz S, Ozgoren E, Sabir N. Comparison of ultrasonographic and anthropometric methods to assess body fat in childhood obesity. Int J Obes. 2007; 31(1):53-8. doi: 10.1038/sj.ijo.0803414.

11. Freedman DS, Thornton JC, Mei Z, Wang J, Dietz WH, Pierson RN, et al. Height and adiposity among children. Obes Res. 2004; 12(5):846-53.

12. Chiara V, Sichieri R, Martins PD. Sensibilidade e especificidade de classificação de sobrepeso em adolescentes, Rio de Janeiro. Rev Saúde Pública. 2003; 37(2):226-31. doi: 10,1590/S0034-891020 03000200010 .

13. Vitolo MR, Campagnolo PDB, Barros ME, Gama CM, Lopez FA. Avaliação de duas classificações para excesso de peso em adolescentes brasileiros. Rev Saúde Pública. 2007; 41(4):653-6. doi: 10.1590/S0034-89102007000400021.

14. Vieira ACR, Alvarez MM, Marins VMR, Sichieri R, Veiga GV. Desempenho de pontos de corte do índice de massa corporal de diferentes referências na predição de gordura corporal em adolescentes. Cad Saúde Pública. 2006; 22(8):1681-1690. doi: 10.1590/S0102-311X2006000800016.

15. Veiga GV, Dias PC, Anjos LA. A comparison of distribution curves of body mass from Brazil and the United States for assessing overweight and obesity in Brazilian adolescents. Rev Panam Salud Publica. 2001; 10(2):79-85. doi: 10.1590/\$1020-49 892001000800002.

16. Sardinha LB, Going SB, Teixeira PJ, Lohman TG. Receiver operating characteristic analysis skinfold thickness and arm girth for obesity screening in children and adolescents. Am J Clin Nutr. 1999; 70(6):1090-5.

17. Maynard LM, Wisemandle W, Roche AF, Chumlea WC, Guo SS, Siervogel RM. Childhood body composition in relation to body mass index. Pediatrics. 2001; 107(2):344-50.

18. Widhalm K, Schonegger K, Huemer C, Auterith A. Does the BMI reflect body fat in obese children and adolescents? A study using the TOBEC method. Int J Obes. 2001, 25(2):279-85.

19. Demerath EW, Schubert CM, Maynard LM, Sun SS, Chumlea WC, Pickoff A, et al. Do changes in body mass index percentile reflect changes in body composition in children? Data from the fels longitudinal study. Pediatrics. 2006; 117(3):487-95. doi: 10.1542/peds.2005-0572.
20. Anjos LA, Veiga GV, Castro IRR. Distribuição dos valores do índice de massa corporal da população brasileira até 25 anos de idade. Rev Panam Salud Publica. 1998; 3(3):164-73. doi: 10.1590/\$1020-498 91998000300004.

21. Luiz RR, Magnanini MMF. A lógica da determinação do tamanho da amostra em investigações epidemiológicas. Cad Saúde Coletiva. 2000; 8(2):9-28.

22. Hallal PC, Bertoldi AD, Gonçalves H, Victora CG. Prevalência de sedentarismo e fatores associados em adolescentes de 10-12 anos de idade. Cad Saúde Pública. 2006; 22(6):1277-87. doi: 10.1590/ S0102-311X2006000600017.

23. Lohman TG, Roche AF, Martorell R. Anthropometric standardization reference manual. Champaign (Illinois): Human Kinetics Books; 1988.

24. Pederson D, Gore C. Erros de medição em antropometria. In: Kevin N, Tim O. Antropométrica. Porto Alegre: Artmed; 2005.

25. Boileau RA, Lohman TG, Slaughter MH. Exercise and body composition in children and youth. Scand J Sports Sci. 1985; 7(1):17-27.

26. Williams DP, Going SB, Lohman TG, Harsha DW, Srinivasan SR, Webber LS, et al. Body fatness and risk for elevated blood pressure, total cholesterol, and serum lipoprotein rations in children and adolescents. Am J Public Health. 1992; 82(3):358-63.

27. Onis M, Onyango AW, Borghi E, Siyam A, Nishida C, Siekmann J. Development of a WHO growth referente for school-aged children and adolescents. Bull World Health Org. 2007; 85(9): 660-7.

28. Farias Júnior JC, Konrad LM, Rabacow FM, Graup S, Araújo VC. Sensibilidade e especificidade de critérios de classificação do índice de massa corporal em adolescentes. Rev Saúde Pública. 2009; 43(1):53-9. doi: 10.1590/50034-89102009 000100007.

29. Fernandes RA, Rosa CSC, Silva CB, Bueno DR, Oliveira AR, Freitas Júnior IF. Desempenho de diferentes valores críticos de índice de massa corporal na identificação de excesso de gordura corporal e obesidade abdominal em adolescentes. Rev Assoc Med Bras. 2007; 53(6):515-9. doi: 10.1590/S0104-42302007000600019.

30. Ramirez Erik, Grijalva-Haro MI, Ponce JA, Valencia ME. Prevalencia de sobrepeso y obesidad en el noroeste de México por tres referencias de índice de masa corporal: diferencias en la clasificación. ALAN. 2006; 56(3):251-6. doi: 10.1590/S0004-0622200 6000300007

Recebido em: 14/3/2008

Versão final reapresentada em: 5/6/2009 Aprovado em: 6/10/2009 
